\title{
ESTUDO DO DESEMPENHO DE UM ANALISADOR DE MOBILIDADE ELÉTRICA DE NANOPARTÍCULAS
}

\author{
E.C.M.CAMARGO, D.M. NUNES, A. C.COSTA, J.J.R. DAMASCENO e F.O.AROUCA
}

Universidade Federal de Uberlândia, Faculdade de Engenharia Química

E-mail para contato: eduarda_eq@yahoo.com.br

\begin{abstract}
RESUMO - Analisador de Mobilidade Elétrica (DMA) consiste em um equipamento utilizado para classificar nanopartículas polidispersas de aerossóis de acordo com a mobilidade elétrica das mesmas, produzindo correntes monodispersas. Tubos concêntricos estão dispostos no sistema de maneira que um eletrodo central é energizado por uma fonte de alta tensão ao passo que um cilindro externo permanece aterrado, produzindo um campo elétrico na região anular. A geração de nanoaerossóis monodispersos é de grande importância, dentre outros aspectos, para a calibração de equipamentos de alta precisão e testes de padronização de filtros de alta eficiência. O presente trabalho consistiu em avaliar o desempenho de um DMA projetado e construído para esta finalidade, utilizando-se de cinco concentrações distintas de $\mathrm{NaCl}$ para análise. Os resultados obtidos mostraram a influência da concentração de sal na quantidade de nanopartículas presentes no aerossol de entrada, sendo que os resultados encontrados para as correntes de aerossol monodisperso e de excesso mostraramse promissores, da mesma forma que o balanço proposto foi bem representativo.
\end{abstract}

\section{INTRODUÇÃO}

Com a intensa industrialização, a geração de efluentes e resíduos sólidos aumentou drasticamente nas últimas décadas, sendo de suma importância gerir de maneira mais sustentável os processos de transformação, o que tem garantido a diminuição de impactos e a redução da emissão de materiais particulados na natureza.Quando se fala em material particulado no ambiente, logo surge a definição de aerossol. Aerossóis são constituídos basicamente por partículas suspensas em correntes gasosas. Quando são encontrados na faixa nanométrica são formados por processos químicos, físicos e/ou biológicos. A importância das nanopartículas para aplicações tecnológicas é crescente nos últimos anos, sendo encontradas em diversos campos, incluindo materiais de síntese, biotecnologia, produção de semicondutores, produtos farmacêuticos, nano- compósitos e cerâmicos.

A classificação eletrostática de aerossóis é o processo pelo qual as partículas de aerossol são separadas em classes de acordo com a sua mobilidade elétrica. Segundo Knutson e Whitby (1975), esta classificação de aerosol tem uma longa história de aplicações, começando com os estudo de eletricidade atmosférica entre meados dos séculos XIX e XX.Foi nos anos 60 que a técnica foi usada para analisar as distribuições de tamanho de aerossóis e os primeiros analisadores de mobilidade foram comercializados (exemplo: Analisador de Aerossol Whitby) (Whitby e Clark, 1966). 
Um parâmetro importante para a caracterização física do comportamento das nanopartículas é o tamanho das mesmas. Entre os vários métodos desenvolvidos para a análise de partículas de aerossol nanométrico, o Analisador de Mobilidade Diferencial (DMA - DifferentialMobilityAnalyzer), tornou-se um instrumento amplamente utilizado para classificar e gerar partículas monodispersas, ou seja, partículas com estreita faixa de diâmetros(INTRA; TIPPAYAWONG, 2008).O importante de se produzir aerossóis monodispersos é calibrar equipamentos de alta precisão devido a ampla variedade de produtos que estão sendo disponibilizados no mercado e que apresentam em sua composição nanopartículas de diferentes composições químicas. Outro fator relevante está diretamente relacionadoà questão de saúde ocupacional, na qual nanopartículas podem oferecer risco ao ser humano enquanto presentes no ar respirável em uma concentração acima da permitida pela legislação ambiental, pois estas podem penetrar no sistema respiratório, sendo de difícil excreção.

O DMA trata-se de um equipamento no qual é aplicada uma tensão no cilindro interno enquanto o cilindro externo é aterrado, o que resulta em um campo elétrico radial. Após as partículas passarem pelo neutralizador, estas apresentarão uma distribuição de carga conhecida, assim a trajetória descrita por uma partícula de aerossol será função apenas do seu diâmetro. O DMA pode então selecionar, em um fluxo de ar com partículas de aerossol de tamanhos diversos (fluxo polidisperso), partículas como função do tamanho e de sua mobilidade elétrica. O objetivo deste projeto é produzir nanopartículas monodispersas em correntes gasosas utilizando-se um equipamento projetado e construído na FEQUI/UFU com o intuito depromover a separação de nanopartículas em aerossóis polidispersos.

\section{TEORIA}

\subsection{Componentes da velocidade da partícula}

As equações diferenciais para a trajetória das partículas dentro do analisador de mobilidade são formuladas em termos de coordenadas cilindricas r(velocidade radial) e $\mathrm{z}$ (velocidade axial), como se segue:

- Velocidade Axial: Considerand্̄ como sendo a velocidade axial média da partícula (suposta como sendo a mesma do gás), pode-se expressá-lapela Equação (1):

$$
\bar{u}=\frac{Q}{\pi\left(r_{2}^{2}-r_{1}^{2}\right)}
$$

sendo Q a vazão volumétrica, A a área da seção transversal do DMA (região anular), $\mathrm{r}_{2}$ o raio do cilindro externo e $\mathrm{r}_{1}$ o raio do cilindro interno.

- Velocidade Radial: Considerando $v_{\text {te }}$ como a velocidade radial da partícula, tem-se a Equação (2):

$$
v_{t e}=E Z_{p}
$$

em que $\mathrm{E}$ a intensidade do campo elétrico para cilindros concêntricos, sendo função da tensão elétrica aplicada (V) e $Z_{p}$ a mobilidade elétrica da partícula, representada pela Equação (3): 


$$
Z_{p}=\frac{\eta e C}{3 \pi \text { 国 } D_{p}}=\frac{Q \ln \left(\frac{r_{2}}{r_{1}}\right)}{2 V L \pi}
$$

Dado $\eta$ igual ao número de cargas; $e$ a carga do elétron $=1,6 \times 10^{-19}$ Coulomb; $\mathrm{C}$ é o Fator de Escorregamento de Cunningham (correção da descontinuidade do meio - adimensional), $\mu$ a viscosidade do gás (aerossol) e $\mathrm{D}_{\mathrm{p}} \mathrm{o}$ diâmetro da partícula.

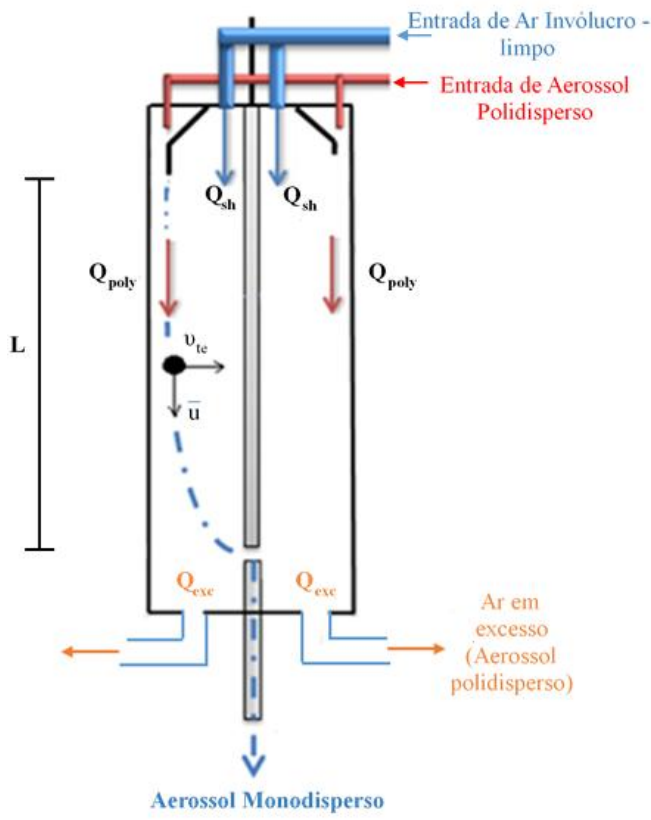

Figura 1 - Distribuição de vazões dentro do DMA

\subsection{Cálculo do diâmetro das partículas presentes no aerossol monodisperso}

Como mostrado na Figura 1, o Analisador de Mobilidade Diferencial apresenta quatro vazões, sendo duas de entrada e duas de saída. As vazões de entradasão chamadas de vazão de Aerossol Polidisperso $\left(\mathrm{Q}_{\text {poly }}\right)$, que adentra o DMA próximo à parede interna do cilindro externo, enquanto que a outra, denominada de vazão de Invólucro ou Diluição $\left(\mathrm{Q}_{\mathrm{sh}}\right)$, penetra o analisador ao redor do cilindro interno. As vazões de saída são denominadas de vazão de Monodisperso $\left(\mathrm{Q}_{\mathrm{mono}}\right)$, ou do aerossol classificado, e vazão de Excesso $\left(\mathrm{Q}_{\mathrm{exc}}\right)$ - corrente constituída pelas partículas não classificadas na fenda de amostragem (ou de classificação).

Sendo o subscrito 1 para os limites inferiores e o subscrito 2 para os limites superiores da banda de mobilidade elétrica, rearranjando as equações de $Z_{p}$, obtém-se:

$$
D_{p}=D_{p}^{*} \frac{C}{C^{*}} \frac{1}{\left(1+\frac{Q_{\text {mono }}}{Q_{s h}}\right)}
$$

em que $D_{p}^{*}$ e $C^{*}$ são os valores do diâmetro da partícula e Fator de Escorregamento de Cunningham centrais, respectivamente. 


\section{MATERIAIS E MÉTODOS}

\subsection{Unidade Experimental}

Com o intuito de verificar a eficiência do classificador de partículas, utilizou-se os seguintes equipamentos na unidade experimental:

- Contador de partículas: utilizado para se obter a concentração de partículas tanto na entrada como na saída do DMA;

- Compressor de ar: para a diluição das partículas nanométricas dos testes experimentais;

- Filtros de ar de alta eficiência: para se obter uma alta purificação de ar;

- Atomizador comercial: utilizado para a geração de aerossol polidisperso uniforme;

- Secador de difusão: usado para remover o excesso de umidade presente no fluxo do aerossol proveniente do atomizador, sendo preenchido com sílica-gel;

- Fonte neutralizadora de raio-X: usado com a finalidade de produzir íons de ar positivos e negativos;

- Rotâmetros: ajuste das vazões de escoamento do gás;

- Mini compressor de ar: finalidade de promover um incremento na vazão e pressão de entrada do aerossol no DMA;

- Fonte de alta tensão: tinha como função estabelecer uma tensão variável no DMA;

- Analisador de Mobilidade Diferencial (DMA): Este equipamento foi projetado e construído na FEQUI/UFU com a finalidade de separar nanopartículas polidispersas produzindo uma corrente de saída monodispersa.

\subsection{Procedimento Experimental}

Soluções aquosas de Cloreto de Sódio foram preparadas nas concentrações de 0,$1 ; 0,5$; 1; 2 e $3 \mathrm{~g} / \mathrm{L}$, utilizando-se água ultrapura como solvente. As concentrações usadas não foram maiores que $3 \mathrm{~g} / \mathrm{L}$ em virtude da formação de aglomerados e da possível corrosão que o sal, em elevadas concentrações, poderia ocasionar nos equipamentos e tubulações.

Os testes iniciavam-se com a purgação do compressor para a retirada de excesso de água com a finalidade de minimizar a umidade do ar e aumentar a vida útil dos filtros de purificação da corrente gasosa e do secador de difusão com sílica em gel. Em seguida, direcionava ar para os filtros que eram interligados ao atomizador comercial contendo um reservatório que era preenchido com a solução de $\mathrm{NaCl}(\approx 80 \mathrm{~mL})$.

Em seguida, as partículas adentravam uma fonte de raio $\mathrm{X}$ com o objetivo de serem neutralizadas, assim o aerossol neutralizado seguia para o Analisador de Mobilidade Elétrica. O DMA estava conectado a uma fonte de alta tensão em que aplicava-se voltagens de 0 $4000 \mathrm{~V}$, sendo que dividiu-se a aplicação de tensão em faixas, totalizando 45 valores de tensão aplicada. As leituras de vazão de entrada, monodisperso e de excesso foram feitas em intervalos de $1 \mathrm{~min}$ durante 10 s utilizando-se o contador de partículas - CPC. Os experimentos foram realizados em treplicadas para se garantir a precisão dos resultados.

Para avaliar a estabilidade na geração de aerossóis, ou seja, do comportamento da geração do atomizador comercial, foram coletadasas concentrações de partículas na entrada 
do DMA para cada concentração de $\mathrm{NaCl}$ usada. A razão $\mathrm{Q}_{\text {poly }}=\mathrm{Q}_{\mathrm{sh}}$ utilizada no DMA foi de 0,6/6 L/min e a pressão no atomizador foi fixada em $103 \mathrm{kPa}$, mantidas nesses valores em todos os testes realizados.

\section{RESULTADOS E DISCUSSÃO}

Para se projetar o DMA da FEQUI/UFU foi necessário primeiramente uma simulação do comportamento do mesmo; para tal, fez-se tratamento estatístico para descobrir as variáveis significativas que influenciariam nos cálculos, sendo estas: as 4 vazões mássicas (ar de invólucro, polidisperso, monodisperso e excesso); o comprimento (L); os diâmetros do cilindro externo e interno: $r_{2}$ e $r_{1}$, respectivamente.

Era necessário encontrar a relação entre tensão e diâmetro de partícula. No cálculo do projeto, simulou-se tensões variando de 20 a $10000 \mathrm{~V}$ e para cada valor detensão foram calculados: $D_{p}^{*} ; C^{*} ; \quad D_{p 1}$ e $D_{p 2} ; \mathrm{C}_{1}$ e $C_{2}$. Como $\mathrm{D}_{\mathrm{p}}$ é função de $\mathrm{C}$ e vice-versa, foi necessário um procedimento iterativo para encontrar os valores de interesse; assim, considerou-se primeiramente $C=1$, sendo necessárias 300 iterações para obter-se um erro inferior a $10^{-6}$. Com os valores da tensão e de $D_{p}^{*}$ encontrados após as iterações, foi possível ajustar $\mathrm{D}_{\mathrm{p}}=f$ (Tensão) utilizando o software tbc $2 \mathrm{D}$, chegando-se à $\mathrm{D}_{\mathrm{p}}=\mathrm{a}+(\mathrm{b} \cdot \mathrm{T})+\left(\mathrm{c} \cdot \mathrm{T}^{2}\right)+\left(\mathrm{d} \cdot \mathrm{T}^{0,5}\right)$ com r ${ }^{2}$ $=0,99999$, dado $\mathrm{a}=1,487904 ; \mathrm{b}=0,025247 ; \mathrm{c}=5,26 \cdot 10^{-7}$ e d = 2,992959.

Da Equação (2.3) é possível calcular a mobilidade elétrica $\left(Z_{\mathrm{p}}\right)$ para cada $D_{p}^{*}$ e $\mathrm{C}^{*}$ calculado, considerandodiferentes números de carga $(\eta)$, usando $\mu=1,83.10^{-5} \mathrm{~kg} / \mathrm{m}$.s; como ilustrado na Figura 2. Pode-se concluir que quanto maior a carga das partículas maior será a mobilidade elétrica apresentada por ela. Outra observação retirada da Figura 2 é a de que quanto maior o tamanho da partícula, menor será a capacidade da mesma em ser atraída pelo campo elétrico, ou seja, menor $Z_{p}$ a partícula terá.

Na Figura 3 apresenta-se a faixa de carregamento adotada no presente estudo, pois trabalhou-se com partículas de diâmetro entre $9 \mathrm{~nm}$ e $\approx 300 \mathrm{~nm}$ (tensão entre 10 e $4000 \mathrm{~V}$ ). É possível notar pela análise da figura que as partículas mais representativas (aquelas que possuem uma probabilidade de carga maior que $20 \%$ ) são aquelas que apresentam $\eta=-1,0$ e +1 .

Para se chegar ao modelo proposto, admitiu-se que: como a saída da fonte de alta tensão é negativa, todas as partículas negativas serão repelidas pelo eletrodo central e ficarão retidas na parede interna do cilindro externo. As partículas neutras e as partículas positivas maiores que $D_{p}^{*}$ estarão presentes na corrente de excesso, enquanto que as partículas positivas de diâmetro $D_{p}^{*}$ serão coletadas na corrente monodispersa e todas as partículas positivas menores do que $D_{p}^{*}$ serão atraídas pelo eletrodocentral. Não há perdas de massa por vazamento nem desprendimento de partículas (rugosidade do tubo >>dp).

Como para cada tensão aplicada ao eletrodo, tem-se uma faixa estreita de diâmetro de partícula que será coletada na fenda de classificação, para o cálculo da concentração de saída no aerossol monodisperso e de excesso, foi realizado um balanço de distribuição de cargas para cada valor de tensão usado. Este balanço considera a entrada total de partículas em cada tensão aplicada, este valor de concentração de entrada é multiplicado pela porcentagem de 
distribuição de cargas para dado diâmetro baseado na distribuição de cargas proposta por Wiedensohler (1988) e representado na Figura 3.

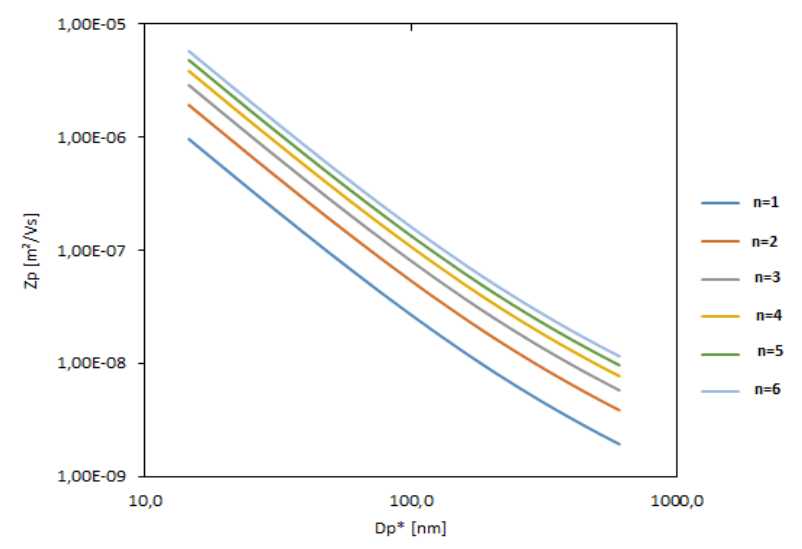

Figura 2 - Mobilidade elétrica das partículas em função do diâmetro para diferentes cargas

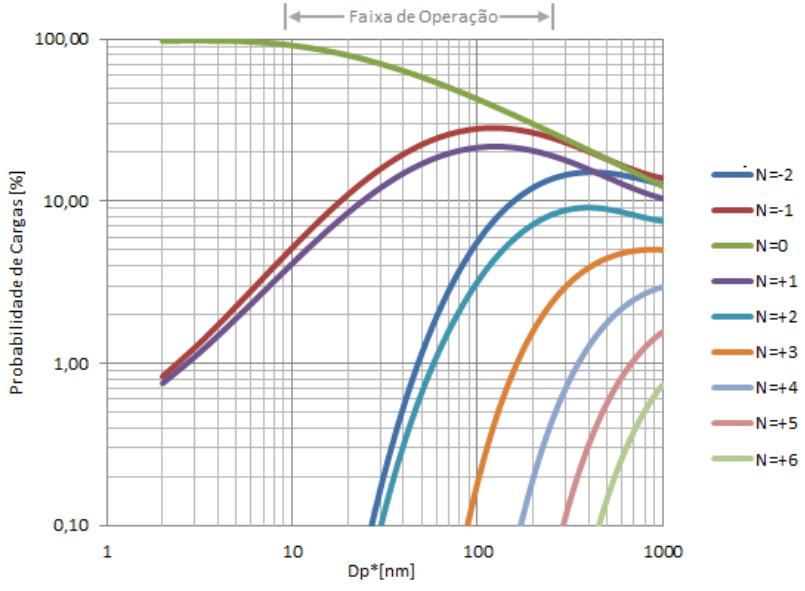

Figura 3 - Distribuição de probabilidades de cargas em função do diâmetro das partículas

Na Figura 4 é mostrado o perfil gráfico esperado da concentração de partículas no aerossol monodisperso e no excesso, utilizando-se o modelo de distribuição de cargas.
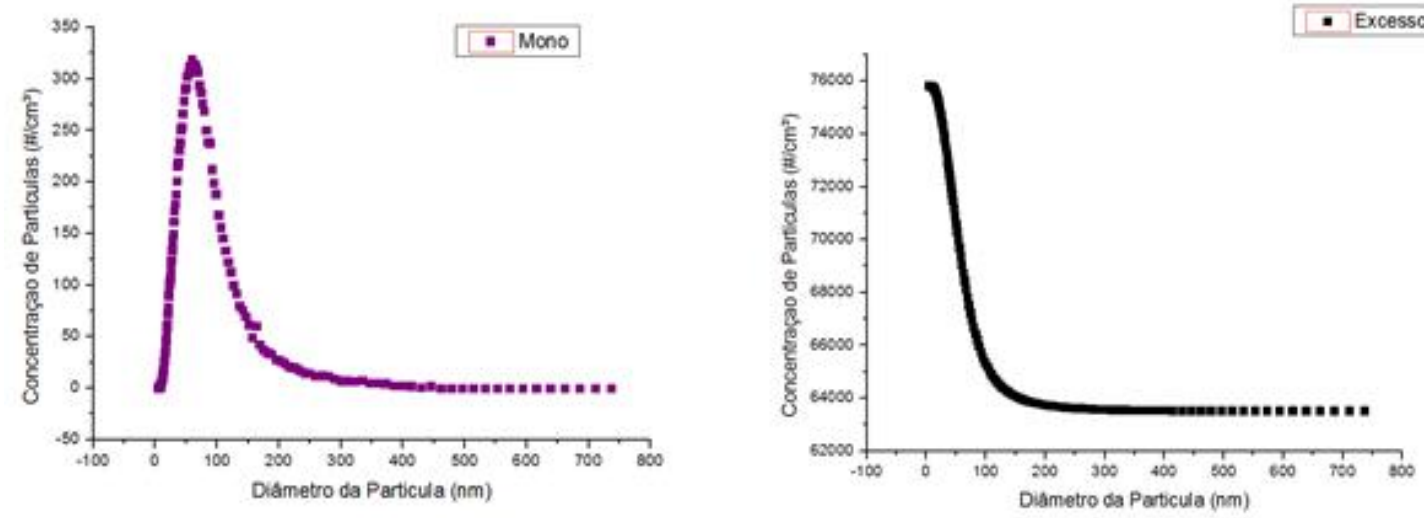

Figura 4 - Modelo proposto pela distribuição de cargas para o Aerossol Monodisperso e de Excesso

Na Figura 5 é possível notar que há uma dependência da concentração de partículas na entrada do DMA com a concentração de $\mathrm{NaCl}$ utilizada na obtenção da solução salina, ou seja, quanto maior a concentração de sal, maior será a concentração de partículas no aerossol formado. Pela análise da Figura 4.4, utilizando a solução de $3 \mathrm{~g} / \mathrm{L}$, tem-se em média 2,65.10 $\# / \mathrm{cm}^{3}$; e para a solução de $0,1 \mathrm{~g} / \mathrm{L}$, há aproximadamente $1,75.10^{5} \# / \mathrm{cm}^{3}$ no aerossol formado, sendo que o símbolo: \# representa onúmero de partículas. Outra observação a ser feita da Figura 4.4 é de que a alimentação do DMA é praticamente constante durante otempo de duração dos testes, o que comprova a eficiência do atomizadorempregado na geração de nanopartículas. 


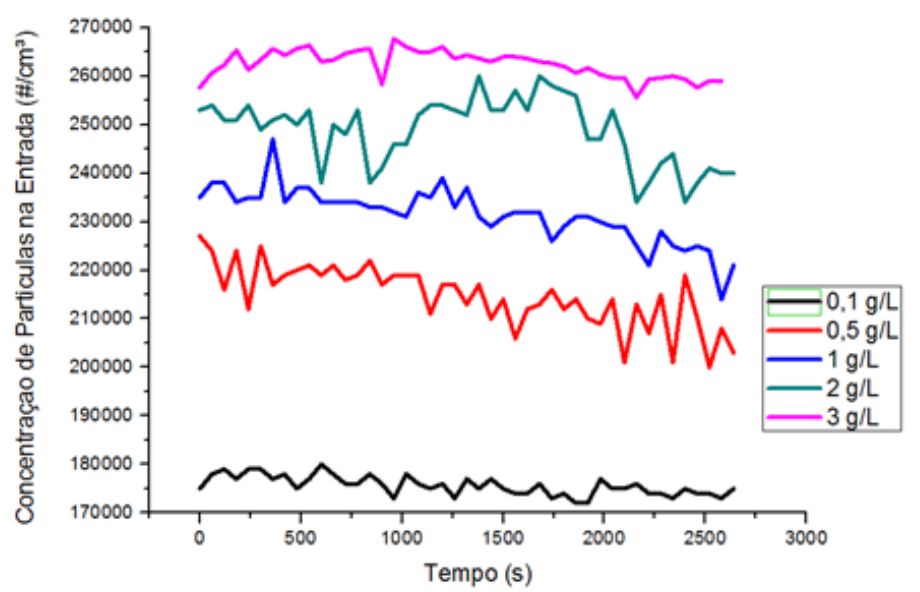

Figura 5 - Comportamento da solução de entrada em função do tempo para as concentrações estudadas

Na Figura 6, estão os gráficos obtidos após a realização das tréplicas para cada concentração estudada neste trabalho, utilizando-se o software Origin para a construção dos mesmos.Nos três primeiros gráficos da Figura 6 está representado o comportamento do aerossol monodisperso e de excesso para as concentrações de 0,$1 ; 2$ e $3 \mathrm{~g} / \mathrm{L}$. Nota-se que o comportamento encontrado era o esperado pelo modelo proposto tanto para a curva de excesso, quanto para a curva de aerossol de saída monodisperso. Este comportamento foi o mesmo encontrado para as soluções de 0,5 e 1 g/L.No quarto gráfico da Figura 6 está representado o comportamento encontrado na vazão monodispersa para as 5 concentrações de $\mathrm{NaCl}$ usadas neste trabalho.Conforme citado por Dalcin (2013), tem-se pela observação do gráfico que com o aumento da concentração de solução salina, os picos de distribuição de tamanhode partículas são mais altos, isto ocorre em virtude da maior concentração de nanopartículasno aerossol.

\section{CONCLUSÕES}

Nos testes realizados com o DMA notou-se quea concentração de partículas aumenta com o aumento da concentração salina utilizada. Além disso, foi possível constatar que o atomizador comercial produz uma concentração uniforme de nanopartículascom o tempo e que os testes com as 5 concentrações de nanopartículas polidispersas foram promissores, mostrando a capacidade de classificação de nanopartículas deste equipamento, produzindo uma corrente de aerossol monodisperso. O balanço de distribuição de cargas para as correntes monodispersas e de excesso foi representativo, porém testes posteriores são necessários para se confirmar o quão representativo o balanço proposto é.

\section{AGRADECIMENTOS}

Agradecemos à Fundação de Amparo à Pesquisa do Estado de Minas Gerais (FAPEMIG) pelos recursos concedidos para a elaboração do projeto e à CAPES pela bolsa de mestrado disponibilizada. 

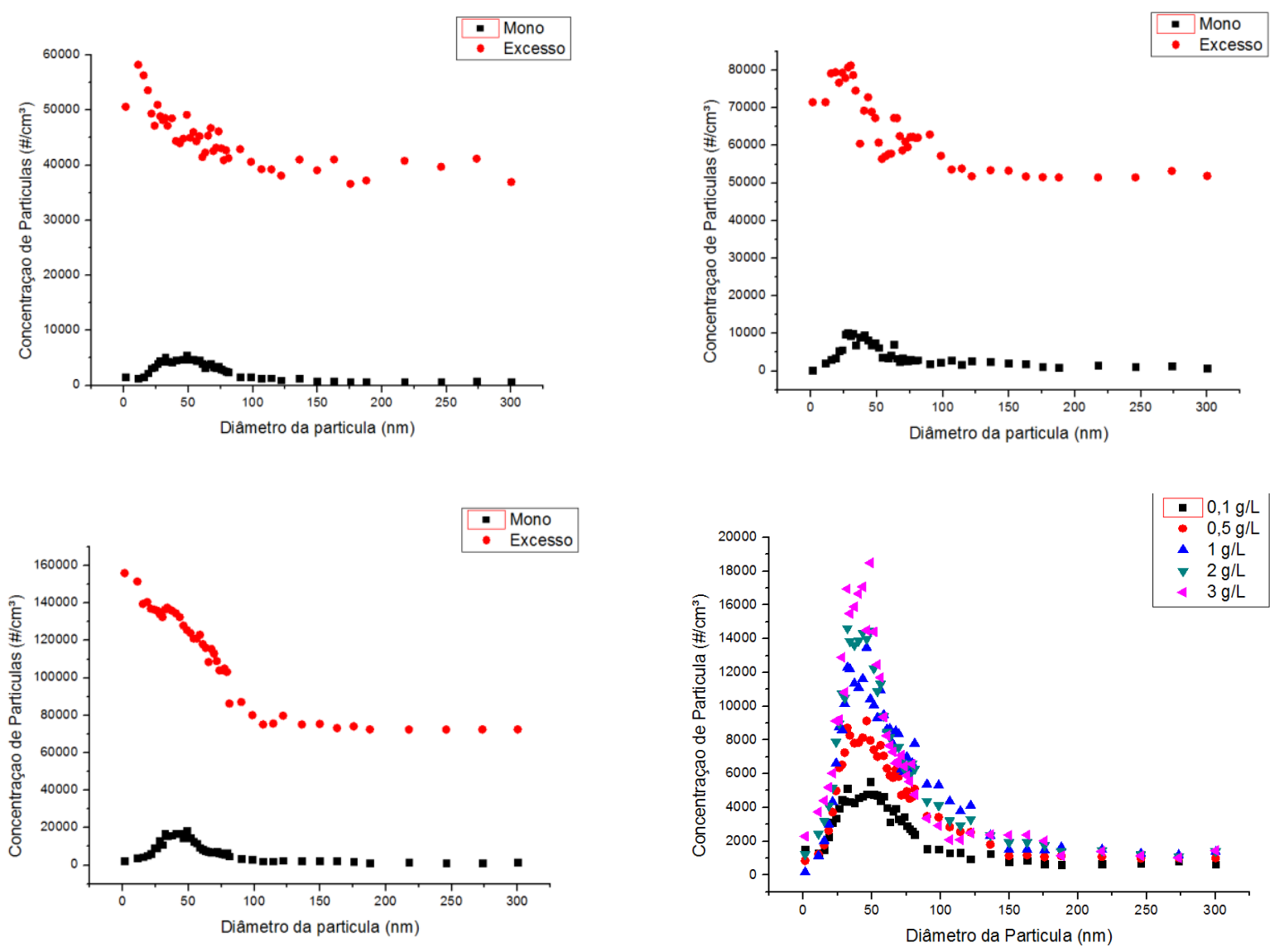

Figura 6 - Comportamento das Concentrações de Aerossol Monodisperso e de Excesso para as concentrações de 0,1; 2 e 3 g/L, respectivamente e Curva do Aerossol Monodisperso para as 5 diferentes concentrações de $\mathrm{NaCl}$ usadas neste trabalho.

\section{REFERÊNCIAS BIBLIOGRÁFICAS}

DALCIN, M.G. Geração de nanopartículas monodispersas em correntes gasosas. Universidade Federal de Uberlândia, 2013, Brasil. Tese de Doutorado.

INTRA, P.; TIPPAYAWONG, N. Analysisofbrowniandifusioneffectonnanometer aero- sol classification in electricalmobilityspectrometer. Journal of the Chinese Institute of Chemical Engineering, 2008.

KNUTSON, E.; WHITBY, K. Aerosol classification by electric mobility: apparatus, theory, and applications. Journal of Aerosol Science, v. 6, p. 443- 451, 1975.

WHITBY, K. T.; CLARK. Electric aerosol particle counting and size distribution measuring system for the 0.015 to 1 um size range. Tellus, v. 18, p. 573-586, 1966. 
WIEDENSOHLER, A. An approximation of the bipolar charge distribution for particles in the submicron size range. Journal of Aerosol Science, v. 19, p. 387-389, 1988. 